\title{
High Incidence of Pediatric Delirium in PICU; Time for Action!*
}

\author{
Erwin Ista, RN, PhD \\ Pediatric Intensive Care \\ Department of Pediatric Surgery \\ Erasmus MC University Medical Center Rotterdam - \\ Sophia Children's Hospital; and \\ Nursing Science \\ Department of Internal Medicine \\ Erasmus MC University Medical Center Rotterdam \\ Rotterdam, The Netherlands

\section{Monique van Dijk, PhD \\ Nursing Science \\ Department of Internal Medicine \\ Department of Pediatric Surgery \\ Erasmus MC University Medical Center Rotterdam - \\ Sophia Children's Hospital \\ Rotterdam, The Netherlands} \\ Erasmus MC University Medical Center Rotterdam; and
}

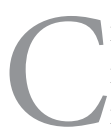

ritically ill patients admitted to an ICU are always "at risk" for acute brain dysfunction, which may manifest itself as reduced consciousness, coma, or delirium (1). The pathophysiology of acute brain dysfunction can be conceptualized as a complex interplay between disease-related factors (e.g., inflammation, severity of illness), predisposing risk factors (e.g., age, cognitive impairment) and environmental factors (e.g., restraint, noise, sleep deprivation, medication such as benzodiazepines) (2). Both adult ICU and PICU survivors may suffer from the postintensive care syndrome in the year after ICU discharge, with mental health problems such as anxiety, depression, and delusional memories (3).

In this issue of Pediatric Critical Care Medicine, Dervan et al (4) present a retrospective single-center study on the risk factors and patient outcomes associated with delirium in a PICU. The prevalence of pediatric delirium (PD) assessed with the Cornell Assessment of Pediatric Delirium (CAPD) among patients with an ICU length of stay of greater than or equal to 48 hours $(n=908)$ was $69 \%$. Multivariable analysis showed that the presence of PD was independently associated with age less than 2 years, baseline cognitive dysfunction, restraint use, mechanical ventilation, benzodiazepine exposure, and low and moderated exposure of dexmedetomidine. Interestingly the duration of

\footnotetext{
*See also p. 21.

Key Words: nonpharmacologic interventions; pediatric delirium; pediatric intensive care unit; prevention; risk factors

The authors have disclosed that they do not have any potential conflicts of interest.

Copyright (C) 2019 by the Society of Critical Care Medicine and the World Federation of Pediatric Intensive and Critical Care Societies

DOI: 10.1097/PCC.0000000000002166
}

delirium was associated with three risk factors that had not previously been described; that is, high dexmedetomidine exposure, days of propofol exposure, and grade III acute kidney injury. The occurrence of PD was not significantly associated with increased in-hospital mortality, in contrast to findings from adult studies and one pediatric study $(5,6)$.

In view of prevention of delirium, the risk factors the authors found, that is, younger age, severity of illness, and cognitive impairment, cannot be influenced. Other risk factors such as benzodiazepines consumption could be tackled, but according to the authors, this is difficult to implement in practice. However, as this has proven feasible in adult ICUs, it would be worthwhile to learn from these experiences (7). Validated clinical decision tools are available, such as the delirium prediction model for ICU patients (ePREDELIRIC), which identify adults at ICU admission for the risk of delirium (8). To our knowledge, such an instrument is not yet available for children. The importance of regular monitoring for delirium with validated assessment tools - such as the CAPD, pediatric pre-school Confusion Assessment - ICU, or the Sophia Observation withdrawal symptoms - Pediatric Delirium Scale (SOSPD scale)—as early as after 48 hours of PICU stay cannot be overemphasized $(9,10)$. Unfortunately, recent surveys revealed that regular monitoring for PD was applied in less than onethird of the participating PICUs $(11,12)$.

The authors found that children under the age of 2 years have a higher probability to develop delirium and also to endure it for a longer time. It must be noted, however, that a recent study found lower inter-rater reliability between nurses applying the CAPD in this age group compared to in older age groups (11). A possible reason is that certain items such as "Does the child communicate needs and wants" may be hard to assess in young children-resulting in contradictory scores between raters. This could have affected the results of the study by Dervan et al (4).

It seems unlikely that pharmacologic treatments can prevent delirium. On the other hand, reducing or even avoiding the use of certain sedatives such as benzodiazepines and propofol seems advantageous and is recommended in the new adult ICU guidelines (13). More complex is the role of dexmedetomidine. The use of alpha- 2 sedatives, including dexmedetomidine, has been promoted, but Dervan et al (4) found that it was associated with the risk of PD. However, they suggest that reverse causality cannot be excluded as dexmedetomidine is also used to control symptoms of delirium.

Nonpharmacologic interventions on the other hand can perhaps help prevent delirium or reduce the severity and duration. Nonpharmacologic interventions are more and more being implemented to provide comfort in critically ill children. Examples are music listening, massage, promoting sleep (e.g., use of earplugs), maintaining a regular day-night cycle, 
avoiding overstimulation by light and sounds, mobilization, and family engagement. Several of these interventions are part of the Assess, Prevent, and Manage Pain, Both Spontaneous Awakening Trials and Spontaneous Breathing Trials, Choice of analgesia and sedation, Delirium: Assess, Prevent, and Manage, Early mobility and Exercise, and Family engagement and empowerment bundle. Early mobilization of adult ICU patients reduced the occurrence of delirium, has been found safe and feasible in children (14). Two ongoing studies on early mobilization in children from research groups in Canada (15) and the United States (16) will show if the results in adults on the occurrence of delirium can be replicated in critically ill children. A recent study on a 24 -hour extended visiting policy in a Brazilian adult ICU reported a reduced delirium prevalence (17). Continuous family presence In the PICU is well accepted in most countries. However, there is a difference between being present or being actively involved. Nurses, child life specialists, and psychologists could tell parents how they can be of help in this regard. They can be encouraged to apply home routines, bring the child's favorite stuffed toy, familiar music, pictures, and toys.

Last, it is well known that delirium in adult ICU patients is associated with long-term consequences such as problems with performing activities of daily life and decline of cognitive functioning (18). The development of a child's brain does not end after birth, but continues into early childhood. Apart from critical illness, also delirium could affect a child's physical, cognitive, emotional, and social health. A recent systematic review shows that children post critical illness are at risk for cognitive impairment (19). Therefore, future research should focus on the long-term consequences of delirium and the implementation of nonpharmacologic interventions.

In summary, PD is a complex manifestation of acute brain dysfunction in the critically ill child and is related to a number of predisposing factors as illustrated by Dervan et al (4) and others. Therefore, the time has come for action in terms of investing in multi-modal interventions for the prevention and treatment of $\mathrm{PD}$ and for follow-up monitoring.

\section{REFERENCES}

1. Tasker RC, Menon DK: Critical care and the brain. JAMA 2016; 315:749-750

2. Meyfroidt G, Smith M: Focus on delirium, sedation and neuro critical care 2019: Towards a more brain-friendly environment? Intensive Care Med 2019; 45:1292-1294
3. Manning JC, Pinto NP, Rennick JE, et al: Conceptualizing post intensive care syndrome in children-the PICS-p framework. Pediatr Crit Care Med 2018; 19:298-300

4. Dervan LA, Di Gennaro JL, Farris RWD, et al: Delirium in a Tertiary PICU: Risk Factors and Outcomes. Pediatr Crit Care Med 2020; 21:21-32

5. Traube C, Silver G, Gerber LM, et al: Delirium and mortality in critically ill children: Epidemiology and outcomes of pediatric delirium. Crit Care Med 2017; 45:891-898

6. Salluh Jl, Wang H, Schneider EB, et al: Outcome of delirium in critically ill patients: Systematic review and meta-analysis. BMJ 2015; $350: \mathrm{h} 2538$

7. Trogrlic $Z$, van der Jagt $M$, Lingsma $H$, et al: Improved guideline adherence and reduced brain dysfunction after a multicenter multifaceted implementation of ICU delirium guidelines in 3,930 patients. Crit Care Med 2019; 47:419-427

8. van den Boogaard M, Schoonhoven L, Maseda E, et al: Recalibration of the delirium prediction model for ICU patients (PRE-DELIRIC): A multinational observational study. Intensive Care Med 2014; 40: 361-399

9. Traube C, Silver G, Kearney J, et al: Cornell assessment of pediatric delirium: A valid, rapid, observational tool for screening delirium in the PICU*. Crit Care Med 2014; 42:656-663

10. Ista $E$, van Beusekom B, van Rosmalen J, et al: Validation of the SOS-PD scale for assessment of pediatric delirium: A multicenter study. Crit Care 2018; 22:309

11. Kudchadkar SR, Yaster M, Punjabi NM: Sedation, sleep promotion, and delirium screening practices in the care of mechanically ventilated children: A wake-up call for the pediatric critical care community*. Crit Care Med 2014; 42:1592-1600

12. Staveski SL, Pickler RH, Lin L, et al: Management of pediatric delirium in pediatric cardiac intensive care patients: An international survey of current practices. Pediatr Crit Care Med 2018; 19:538-543

13. Devlin JW, Skrobik Y, Gélinas C, et al: Clinical practice guidelines for the prevention and management of pain, agitation/sedation, delirium, immobility, and sleep disruption in adult patients in the ICU. Crit Care Med 2018; 46:e825-e873

14. Wieczorek B, Ascenzi J, Kim Y, et al: PICU up!: Impact of a quality improvement intervention to promote early mobilization in critically ill children. Pediatr Crit Care Med 2016; 17:e559-e566

15. Early rehabilitation in critically ill children - The PICU Liber8 Study (PICULiber8). Available at: https://clinicaltrials.gov/ct2/show/ NCT03573479. Accessed October 21, 2019

16. PICU Up!: Trial of a multicomponent early mobility intervention for critically ill children. Available at: https://ClinicalTrials.gov/show/ NCT03860168. Accessed October 21, 2019

17. Westphal GA, Moerschberger MS, Vollmann DD, et al: Effect of a 24-h extended visiting policy on delirium in critically ill patients. Intensive Care Med 2018; 44:968-970

18. Brummel NE, Jackson JC, Pandharipande PP, et al: Delirium in the ICU and subsequent long-term disability among survivors of mechanical ventilation. Crit Care Med 2014; 42:369-377

19. Kachmar AG, Irving SY, Connolly CA, et al: A systematic review of risk factors associated with cognitive impairment after pediatric critical illness. Pediatr Crit Care Med 2018; 19:e164-e171 